\title{
Análisis térmico de soldadura GTAW sobre placa de acero AISI 316L empleando el método de elementos finitos
}

\section{(GTAW welding thermal analysis on AISI 316L steel plate using the finite elements method)}

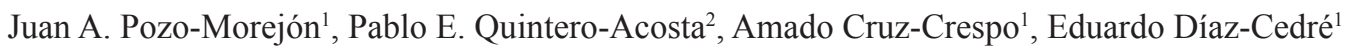

${ }^{1}$ Centro de Investigaciones de Soldadura. Universidad Central "Marta Abreu” de Las Villas. Santa Clara, Villa Clara, Cuba, jpozo@, uclv.edu.cu

${ }^{2}$ Empresa de diseño y servicios de ingeniería UEB Villa Clara, Cuba.

\section{Resumen}

En el presente trabajo se realiza la modelación térmica de soldadura GTAW sobre placa de acero inoxidable AISI 316L. Se analizan los aspectos teóricos más relevantes a considerar durante la ejecución de un análisis de este tipo. En la simulación se emplea un software de análisis por elementos finitos de uso general y se enriquece una metodología, previamente desarrollada, para la modelación en $3 D$ no lineal transitoria del proceso de soldeo. En dicha metodología se implementa una subrutina en lenguaje APDL, programada con el modelo volumétrico de fuente calor de doble elipsoide, en un sistema de coordenadas cartesiano. Se analiza la influencia del paso de tiempo seleccionado sobre los resultados de la simulación. Finalmente se valida la metodología enriquecida, en base a la correlación de los resultados del modelo respecto a los resultados experimentales.

Palabras clave: Soldadura, GTAW, modelación, ANSYS.

Abstract: In the present work, thermal modeling of GTAW welding on AISI $316 \mathrm{~L}$ stainless steel plate is presented. More relevant theoretical aspects to be considered during the implementation of an analysis of this type are discussed. For the simulation a general purpose finite element analysis software has been used. A previously developed methodology for 3D nonlinear transient modeling of welding process has also been improved. In this methodology a subroutine in APDL language is implemented, programmed with the double ellipsoid volumetric heat source model, in a Cartesian coordinated system. The influence of the selected time step on the simulation results is analyzed. The good correlation obtained among the results calculated by means of the model and the experimental data validates this improved methodology.

Key words: Welding, GTAW, modelation, ANSYS.

\section{Introducción}

En las tres últimas décadas se han realizado múltiples investigaciones dirigidas a procedimientos analíticos que simulen con más precisión los procesos de soldadura, empleando el análisis por elementos finitos (AEF). Estas modelaciones se realizan con software de elementos finitos especialmente desarrollados para aplicaciones en soldadura como el WELDSIM [1] o el SYSWELD [2], o mediante software comerciales de uso general como ANSYS [3-5], ABAQUS o COSMOS [6]. El empleo de un software de uso general trae consigo la necesidad de desarrollar metodologías y programas para la ejecución del análisis térmico de soldaduras. De esta manera se requiere implementar las propiedades termodependientes del material, los modelos de fuentes de calor a emplear y su desplazamiento sobre la pieza, el aporte de material, si existiera, las condiciones de frontera, entre otros.

En este contexto, en el presente trabajo se propone el enriquecimiento de una metodología de simulación térmica de

(Recebido em 06/06/2011; Texto final em 30/08/2011). soldaduras, previamente desarrollada por Pozo-Morejón [7,8], que emplea el software ANSYS Multiphysics versión 12.0. En dicha metodología se implementa el modelo volumétrico de fuente de calor más universalmente empleado, modelo de doble elipsoide, mediante una subrutina en lenguaje APDL (ANSYS Parametric Design Language). La metodología se aplica con éxito a la simulación de un experimento de soldadura GTAW sobre placa de acero inoxidable austenítico, llevado a cabo por Depradeux [9].

\section{Fundamentos del análisis térmico de soldaduras mediante elementos finitos}

La ecuación de difusión del calor en la que se basan los programas de AEF para realizar el análisis térmico de una soldadura se expresa de dos maneras $[3,10]$ :

$$
\begin{aligned}
& \rho(T) c(T) \frac{\partial T}{\partial t}=q+\frac{\partial}{\partial x}\left[K x(T) \frac{\partial T}{\partial x}\right]+\frac{\partial}{\partial y}\left[K y(T) \frac{\partial T}{\partial y}\right]+\frac{\partial}{\partial z}\left[K z(T) \frac{\partial T}{\partial z}\right] \\
& \frac{\partial H}{\partial t}=q+\frac{\partial}{\partial x}\left(K_{x}(T) \frac{\partial T}{\partial x}\right)+\frac{\partial}{\partial y}\left(K_{y}(T) \frac{\partial T}{\partial y}\right)+\frac{\partial}{\partial z}\left(K_{z}(T) \frac{\partial T}{\partial z}\right)
\end{aligned}
$$

donde $\rho(T)$ - representa la densidad del material como propiedad termo dependiente, $\mathrm{c}(\mathrm{T})$ - calor específico, $\mathrm{H}$ - entalpía específica del material, q - calor generado por unidad de volumen, $\mathrm{Kx}(\mathrm{T})$, 
$\mathrm{Ky}(\mathrm{T}), \mathrm{Kz}(\mathrm{T})$ - coeficientes de conductividad térmica en las tres direcciones, $\mathrm{T}$ - temperatura y $\mathrm{t}$ - tiempo.

\subsection{Modelo de fuente de calor de doble elipsoide}

El modelo de fuente de calor de doble elipsoide de Goldak constituye uno de los más universalmente empleados en la simulación de soldaduras, ya que se adapta a procesos de soldadura por arco, láser y haz de electrones, entre otros [11]. Este modelo combina dos fuentes, de manera que la mitad delantera está constituida por el cuadrante de una fuente elipsoidal y la mitad trasera por el cuadrante de otra (figura 1). Dicho modelo matemático permite realizar el cálculo de la densidad de calor, o densidad de potencia térmica, que actúa en cada punto de interés que se encuentre dentro del volumen de la fuente (zona gris de figura), en un sistema de coordenadas cartesiano, a partir de las ecuaciones que a continuación se muestran $[11,12]$.

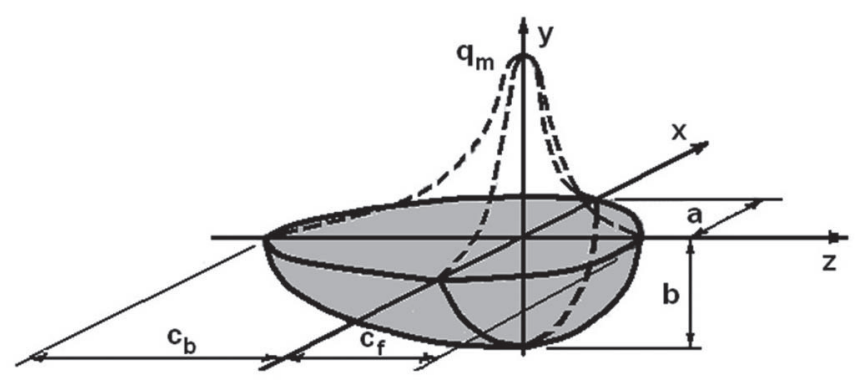

Figura 1 - Modelo de fuente de calor de doble elipsoide.

Para los puntos que se encuentren dentro del cuarto de elipsoide que va al frente, la ecuación de la densidad de potencia térmica es la siguiente:

$q(x, y, z)=\frac{6 \sqrt{3} \cdot r_{f} \cdot Q}{a b c_{f} \pi \sqrt{\pi}} \exp \left(-\frac{3 x^{2}}{a^{2}}-\frac{3 y^{2}}{b^{2}}-\frac{3 z^{2}}{c_{f}^{2}}\right) \quad\left(\mathrm{W} / \mathrm{m}^{3}\right)$

Para los puntos que se encuentren dentro del cuarto de elipsoide trasero:

$q(x, y, z)=\frac{6 \sqrt{3} \cdot r_{b} \cdot Q}{a b c_{b} \pi \sqrt{\pi}} \exp \left(-\frac{3 x^{2}}{a^{2}}-\frac{3 y^{2}}{b^{2}}-\frac{3 z^{2}}{c_{b}^{2}}\right)$

Donde a, b y c - parámetros dimensionales de la fuente de calor elipsoidal (figura 1) (m), x, y, z- coordenadas sobre cada eje del punto de interés dentro del elipsoide respecto a su centro (m), $\mathrm{Q}$ - potencia térmica efectiva del $\operatorname{arco}(\mathrm{W}), \mathrm{r}_{\mathrm{f}} \mathrm{y}_{\mathrm{b}}$ - coeficientes adimensionales de proporción de aporte del calor en la parte delantera y trasera de la fuente, respectivamente (se tiene que cumplir que $r_{f}+r_{b}=2$ ). Los valores para estos coeficientes se determinan como [17]:

$r_{f}=\frac{2 c_{f}}{\left(c_{f}+c_{b}\right)} \quad r_{b}=\frac{2 c_{b}}{\left(c_{f}+c_{b}\right)}$

Para procesos de soldeo por arco eléctrico:
Donde $\eta$ - representa la eficiencia térmica de la fuente de calor, $\mathrm{V}$ - voltaje de arco (V); I - corriente de soldadura (A).

La densidad de potencia térmica máxima " $\mathrm{q}_{\mathrm{m}}$ " se aplica en el centro del doble elipsoide (figura 1) y equivale a la calculada por la expresión que antecede a la exponencial en las expresiones 3 y 4 . En la medida que el punto de interés dentro del elipsoide se encuentra más alejado de dicho centro la densidad de potencia térmica disminuye de forma Gaussiana. Para cualquier punto fuera de la frontera de los dos cuartos de elipsoide la densidad de potencia térmica que se aplica es nula.

De esta manera la distribución de calor en este modelo se describe por cinco parámetros, uno es la eficiencia del arco $\eta$ y cuatro que son parámetros dimensionales $\mathrm{a}, \mathrm{b}, \mathrm{c}_{\mathrm{f}}, \mathrm{c}_{\mathrm{b}}$. Debido a la equivalencia entre las dimensiones del modelo de fuente y la del baño de soldadura algunos autores sugieren que los valores apropiados para $\mathrm{a}, \mathrm{b}, \mathrm{c}_{\mathrm{f}}, \mathrm{c}_{\mathrm{b}}$ deben obtenerse por medición directa experimental de la geometría de la zona fundida (ZF) de la soldadura (macrografía de su sección transversal y de los rizos de la superficie del baño), donde (a) es el semiancho de la zona fundida, (b) la penetración y $\left(\mathrm{c}_{\mathrm{f}}, \mathrm{c}_{\mathrm{b}}\right)$ el largo delantero y trasero del baño [11]. Esto tiene el inconveniente de que cada régimen de soldadura en particular requeriría realizar varios depósitos y efectuar las mediciones experimentales.

Es importante destacar que para un mismo calor de entrada (Q) si las dimensiones del modelo de doble elipsoide aumentan, disminuyen las dimensiones de la zona fundida y viceversa. Es recomendable calibrar el modelo de doble elipsoide, que finalmente se empleará en las simulaciones, variando los parámetros $\eta, \mathrm{a}, \mathrm{b}, \mathrm{c}_{\mathrm{f}}, \mathrm{c}_{\mathrm{b}}$ hasta ajustar las dimensiones de la zona fundida y las curvas de ciclo térmico, calculadas por el modelo respecto a las determinadas experimentalmente para puntos situados a diferentes distancias del eje de la soldadura.

Goldak establece que si se pretende estudiar en detalle la región cerca del baño este modelo de doble elipsoide puede ser muy favorable, sobre todo combinado con una malla fina con alrededor de diez elementos lineales a lo largo del eje del área elipsoidal de entrada de calor [15]. A pesar de lo anterior, este modelo constituye una simplificación que no considera todos los fenómenos físicos complejos que ocurren dentro de la fuente de calor real y dentro del baño de soldadura, por lo que se debe ser cauteloso cuando la zona de interés es precisamente dicho baño. Fuera de la ZF el modelo garantiza precisión de resultados por lo que en el presente trabajo se hace énfasis en su implementación.

\subsection{Movimiento del modelo de fuente de calor $y$ generalización de su expresión matemática para un sistema de coordenadas cartesiano}

En los modelos de elementos finitos que simulan un proceso de soldeo existe un sistema de coordenadas cartesiano fijo global $(\mathrm{X}, \mathrm{Y}, \mathrm{Z})$ y se declara un sistema móvil $(\mathrm{x}, \mathrm{y}, \mathrm{z})$ que se encuentra ubicado en el centro de la fuente de calor y que se desplaza con esta (figura 2). 


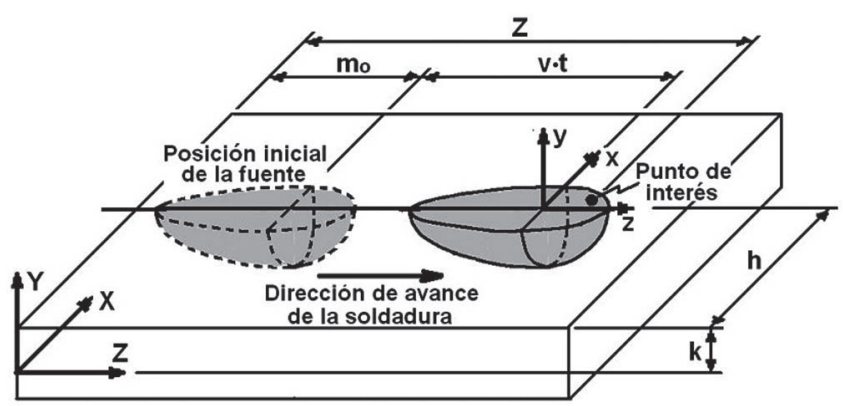

Figura 2 - Sistemas de coordenadas empleados en la modelación.

A modo de ejemplo, para el caso general de una fuente que se desplaza en una dirección paralela al eje de coordenadas " $Z$ " la expresión 3, que define el modelo de fuente, se transforma en:

$q(x, y, z)=\frac{6 \sqrt{3} \cdot r_{f} \cdot Q}{a b c_{f} \pi \sqrt{\pi}} \exp \left(-\frac{3(X-h)^{2}}{a^{2}}-\frac{3(Y-k)^{2}}{b^{2}}-\frac{3\left(Z-m_{o}-v t\right)^{2}}{c_{f}{ }^{2}}\right)$

Donde X, Y, Z - representan las coordenadas del punto de interés dentro del elipsoide respecto al sistema de coordenadas global (m), "h" y "k" - distancias sobre el eje " $\mathrm{X}$ " y "Y" respectivamente entre el origen del sistema de coordenadas fijo y el móvil que se desplaza con la fuente $(\mathrm{m}), \mathrm{m}_{\mathrm{o}}$ - define la posición del centro de la fuente (origen del sistema de coordenadas móvil) en dirección al eje " $Z$ " para $t=0$ respecto al origen del sistema de coordenadas global (téngase en cuenta que $\mathrm{m}_{\mathrm{o}}$ es negativo cuando el centro del sistema coordenado móvil está sobre semieje " $Z$ " negativo, $y$ es positivo en caso contrario) $(\mathrm{m}), \mathrm{v}$ - velocidad de soldeo $(\mathrm{m} / \mathrm{s})$, $\mathrm{t}$ - tiempo transcurrido ( $\mathrm{s}$ ).

De esta manera, en cualquier instante de tiempo, múltiplo del paso de tiempo, los parámetros $\mathrm{h}, \mathrm{k}, \mathrm{m}_{\mathrm{o}}, \mathrm{v}, \mathrm{t}$ definen la posición del origen del sistema móvil de coordenadas (centro de la fuente de calor) respecto al origen del sistema global. Precisamente el producto "vt" es el que define el desplazamiento de la fuente en dirección del eje "Z" para cualquier instante. La expresión 4 se transforma de manera análoga a la 3.

\subsection{Paso de tiempo en el cálculo computacional}

Durante la simulación en estado transitorio de un proceso de soldeo mediante el AEF el modelo de fuente de calor no se traslada de forma continua, sino a intervalos de un paso de tiempo (time-step). De esta manera, el modelo de elementos finitos se convierte no en una soldadura continua, sino conformada por una serie de zonas en las que se aplica el calor de manera secuencial y que generalmente se solapan unas con otras.

En zonas de severos gradientes térmicos (zona cercana a la soldadura) existe una relación entre la dimensión del elemento en la dirección del flujo de calor y el menor paso de tiempo que conduce a buenos resultados. Francis, Cronje, entre otros, recomiendan emplear la expresión 9, con vistas a definir el paso de tiempo mínimo (o máximo tamaño de elemento) $[2,13]$.

Paso de tiempo $\geq \frac{\delta^{2}}{\alpha}$
Donde $\delta$ - dimensión del elemento finito a lo largo de la dirección de conducción del calor, perpendicular al eje de la soldadura (m); $\alpha$ - difusividad térmica del material $\left(\mathrm{m}^{2} / \mathrm{s}\right)$.

Según otras fuentes, cuando se emplea el "paso de tiempo automático" y elementos cuadráticos se recomienda que el paso de tiempo se calcule por expresión similar a la 9 , pero incluyendo un 4 en el denominador $[14,15]$. Ambas fórmulas conducen a resultados de paso de tiempo relativamente pequeños, sobre todo para mallas finas, que de emplearse aumentarían la precisión del resultado, pero también los tiempos de cálculo. De esta manera, se prefiere en el trabajo la expresión 9 que garantiza un paso de tiempo mayor, que a su vez cumple con esta segunda condici ón.

Otra recomendación respecto al paso de tiempo a emplear ha sido formulada por Goldak, quien establece que la fuente de calor debe avanzar aproximadamente la mitad de su longitud para modelos planos tridimensionales, mientras que si avanza más de tres veces su longitud (la aplicación del calor es en zonas aisladas sin solapamiento), entonces la diferencia entre el campo de temperaturas medido y el calculado puede ser grande [11, 13]. En otro trabajo este mismo autor emplea un paso de tiempo inferior, de 1/3 de la longitud del modelo de fuente [16]. Se debe por tanto evitar magnitudes de pasos de tiempo menores que el calculado por la expresión 9 y mayores que un valor aun no precisado con claridad en la literatura, por lo que constituye un tópico importante a estudiar.

\section{Propiedades termofísicas del acero AISI 316L}

En los modelos de transferencia del calor, como se ha mostrado en epígrafe 2, se deben introducir tres propiedades termofísicas básicas del material: conductividad, densidad y calor específico. Sin embargo, en análisis térmicos transitorios no lineales donde se simulan cambios de fase (como en la soldadura), se necesita considerar el calor latente, que es la energía que el sistema almacena o entrega durante dicho cambio [14]; lo anterior se logra declarando como propiedad del material la entalpía en función de la temperatura, que como concepto agrupa a la densidad y el calor específico, uno de los aspectos que enriquecen la metodología de modelación de soldaduras empleada $[7,8]$.

Existen discrepancias entre los autores sobre las temperaturas de sólido y líquido del acero AISI 316L, que determinan el intervalo del calor latente. Tissot considera que estas temperaturas son $1400-1500{ }^{\circ} \mathrm{C}$ respectivamente, mientras que Desroches las establece en 1450 - $1500{ }^{\circ} \mathrm{C}$ [9]. En un inicio fue tomado, para la modelación del proceso de soldeo, como intervalo de calor latente 1450 - $1500{ }^{\circ} \mathrm{C}$, lo cual implica una brusca variación de la entalpía en un intervalo de $50{ }^{\circ} \mathrm{C}$, y provoca dificultades de convergencia al ejecutar los cálculos, corroborando lo establecido por Sun [17]. Tal dificultad fue superada mediante la ampliación del intervalo de calor latente de $1420{ }^{\circ} \mathrm{C}$ a $1500{ }^{\circ} \mathrm{C}$ (rango de $80{ }^{\circ} \mathrm{C}$ ), garantizándose un buen balance entre velocidad de convergencia de la solución y precisión de los resultados.

En el trabajo fue tomada la conductividad térmica 
brindada por Depradeux, y como este autor recomienda, para la temperatura de líquido " $\mathrm{T}_{1}$ " el valor de dicha propiedad fue duplicado para simular la transferencia de calor por convección que tiene lugar en el baño [9]. Por encima de " $T_{1}$ " esta propiedad fue mantenida constante (figura 3 ).

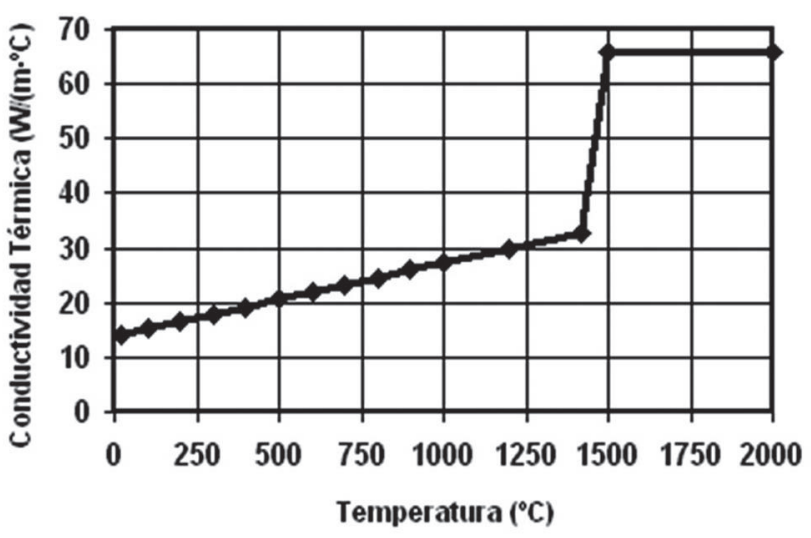

Figura 3 - Conductividad térmica del acero AISI 316L.

Para definir la entalpía se tomaron los valores calculados por Becerra, a partir de la densidad y calor específico dados por Depradeux $[4,9]$. Como recomiendan ambos autores el valor fue extrapolado hasta " $\mathrm{T}$ ", luego la propiedad fue calculada a la temperatura " $\mathrm{T}_{1}$ " $\left(\mathrm{H}_{\mathrm{TI}}=\mathrm{H}_{\mathrm{TS}}+\right.$ Calor latente de fusión), y por encima de " $\mathrm{T}_{1}$ " fue mantenida la pendiente de la curva. Para esta aleación fue asumido como calor latente de fusión $1,8 \cdot 10^{9} \mathrm{~J} / \mathrm{m}^{3}$ [9]. Vea figura 4.

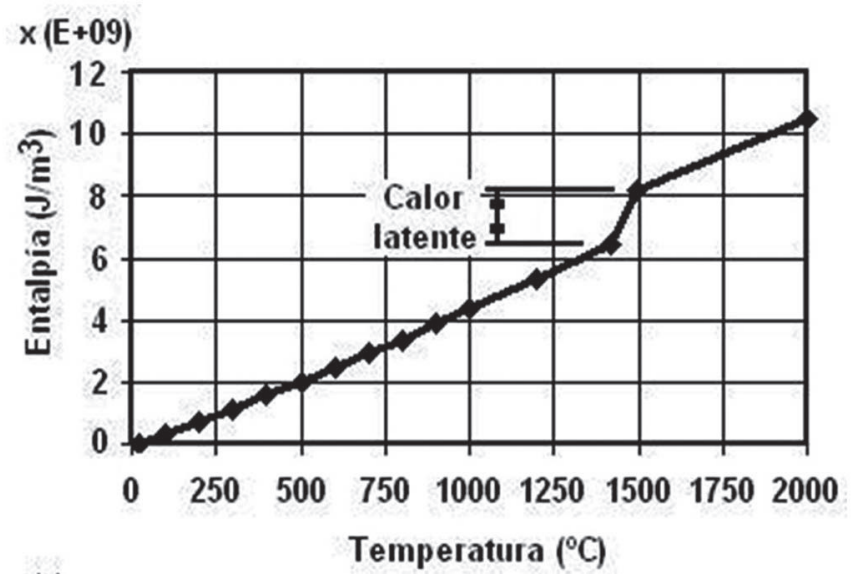

Figura 4 - Entalpía del acero AISI 316L.

Sin dudas, los posibles errores que se introducen al declarar las propiedades termodependientes constituyen una dificultad al ejecutar las simulaciones de soldadura, debido a la diversidad de criterios entre las diferentes fuentes. Debido a esto, con el propósito de garantizar precisión de las simulaciones, dichas propiedades deben ser calibradas en base a los resultados de los modelos y los experimentales.

\section{Análisis térmico mediante elementos finitos de soldadura GTAW sobre placa AISI 316L}

En el presente trabajo se simula un experimento de soldadura realizado por Depradeux [9], sobre la superficie de una placa de acero inoxidable AISI 316L, de $250 \times 160 \times 10 \mathrm{~mm}$. El procedimiento empleado fue: proceso GTAW con $\mathrm{CD}$, argón como gas protector, electrodo de tungsteno - torio, diámetro 1,6 $\mathrm{mm}$ y sin aporte. Los parámetros utilizados en el experimento fueron: corriente de $150 \mathrm{~A}$, tensión de $10 \mathrm{~V}$, velocidad de soldeo de $40 \mathrm{~mm} / \mathrm{min}$ y flujo de gas de 11 litros/min. La longitud del cordón fue de $230 \mathrm{~mm}$, y este comienza y termina a $10 \mathrm{~mm}$ del borde de la placa (vea figura 5 donde se representa la mitad de la placa). La duración de la soldadura fue de $345 \mathrm{~s}$.

Para la validación de las simulaciones serán utilizados los datos experimentales de ciclos térmicos y la macrografía de la zona fundida, obtenidos por Depradeux en una sección transversal, denominada por este como "ST1", a $95 \mathrm{~mm}$ del borde de la placa (figura 5) [9]. Los termopares fueron colocados por este autor sobre la superficie superior de placa a una distancia de 10, 25 y $50 \mathrm{~mm}$ del eje de la soldadura (Figura 5 a) y sobre la inferior a $0,5,10,20$ y $50 \mathrm{~mm}$ (Figura $5 \mathrm{~b}$ ). A partir de dicha macrografía se realizaron mediciones que arrojaron un semiancho de la zona fundida de $3,4 \mathrm{~mm}$ y una penetración de $4,1 \mathrm{~mm}$.

Es importante aclarar que Depradeux en su trabajo, además, modeló el proceso de soldeo en $2 \mathrm{D}$ y $3 \mathrm{D}$, empleando un modelo simplificado de fuente de calor y el software Aster [9].

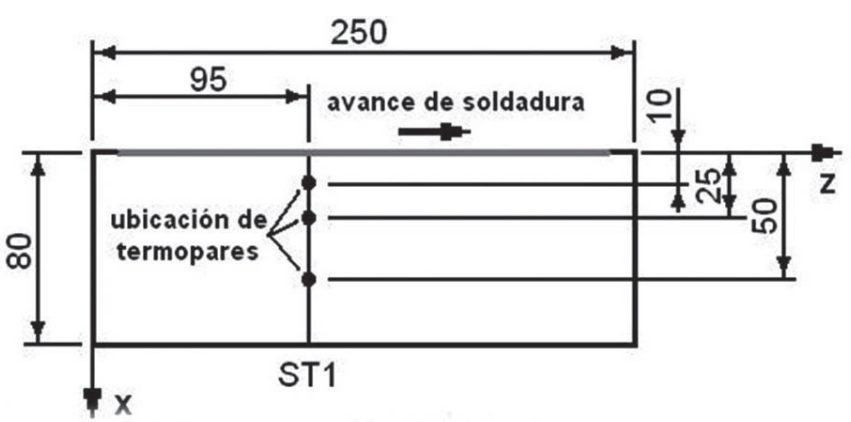

a) Mitad de la cara superior de placa por donde se ejecuta soldadura

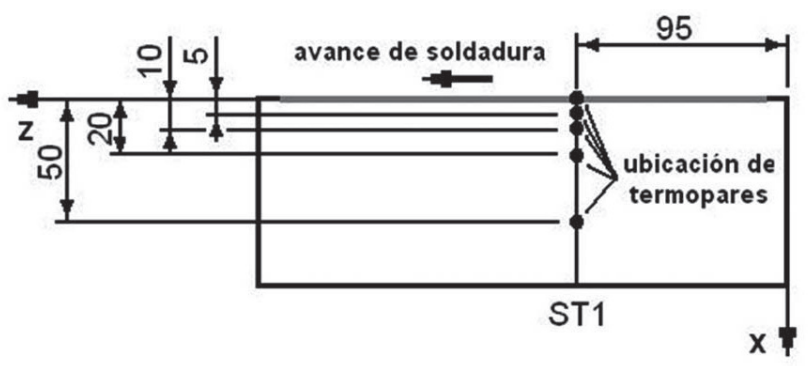

b) Mitad de cara inferior

Figura 5 - Posición de los termopares sobre ambas caras de la placa, en sección transversal ST1. 


\subsection{Mallado del modelo}

Desde el punto de vista de las condiciones de frontera, que incluye la carga térmica que se aplica y la pérdida de calor por convección, así como el proceso de transferencia de calor por conducción a través del sólido, el modelo a analizar es simétrico, por lo que se analiza solo la mitad de la pieza, lo que reduce el tiempo computacional.

El elemento usado en el mallado para el análisis térmico es el SOLID70, un elemento hexaédrico lineal de ocho nodos y un grado de libertad, la temperatura, recomendado para ejecutar análisis no lineales térmicos transitorios [14]. Fue ejecutado un mallado de la pieza más refinado cerca del área de soldadura, donde ocurren los mayores gradientes térmicos, que se va haciendo más basto a medida que se aleja de esta. Fueron utilizadas tres dimensiones de malla, una gruesa con elementos cúbicos en la zona de la soldadura y cercana a esta, con dimensión de arista de $2,5 \mathrm{~mm}$ (con 4200 elementos y 5325 nodos), una media de con dimensión de $1,25 \mathrm{~mm}$ (con 22400 elementos y 24498 nodos) y una fina con $0,625 \mathrm{~mm}$ (con 60000 elementos y 65070 nodos) (figuras 6,7 y 8 ). Para el caso de la malla fina esta dimensión de elemento fue determinada a partir del criterio de que garantizara al menos cinco elementos dentro de la dimensión del semiancho del elipsoide "a" (figura 1), que se hizo coincidir aproximadamente con el semiancho de la ZF determinado experimentalmente, concordando con recomendación de Goldak, ya referida en epígrafe 2.1.

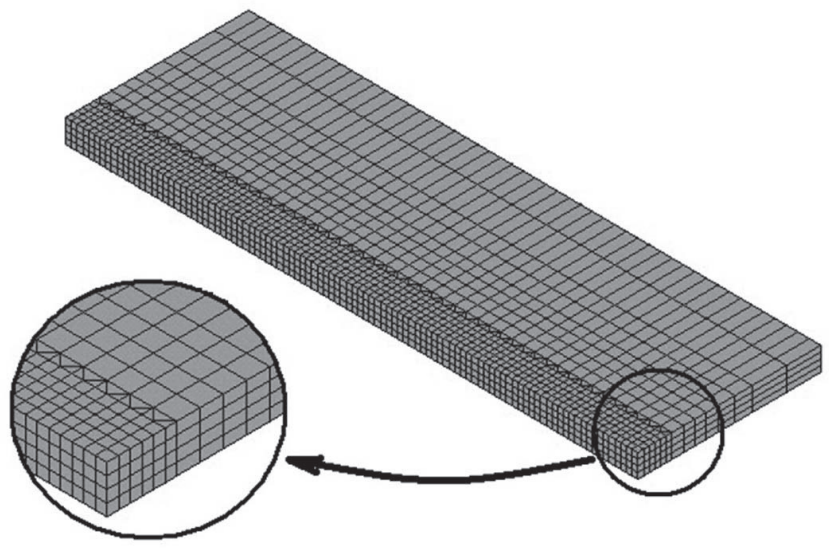

Figura 6 - Malla gruesa.

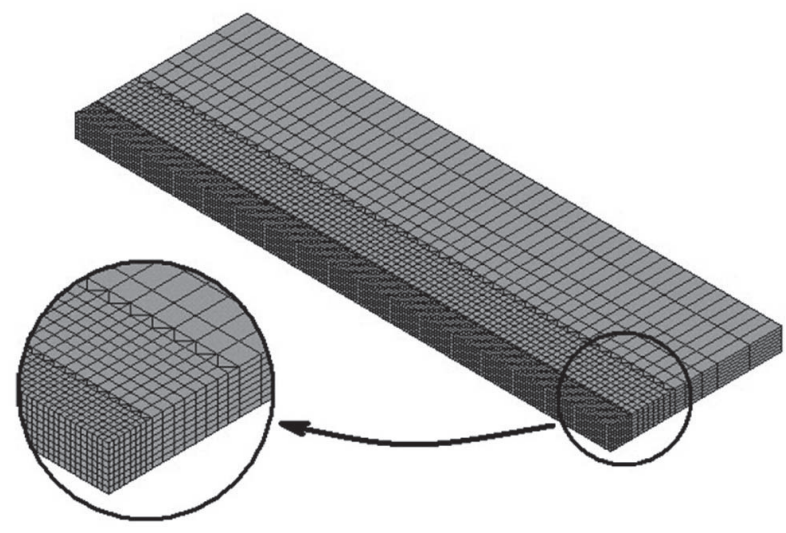

Figura 7 - Malla media.

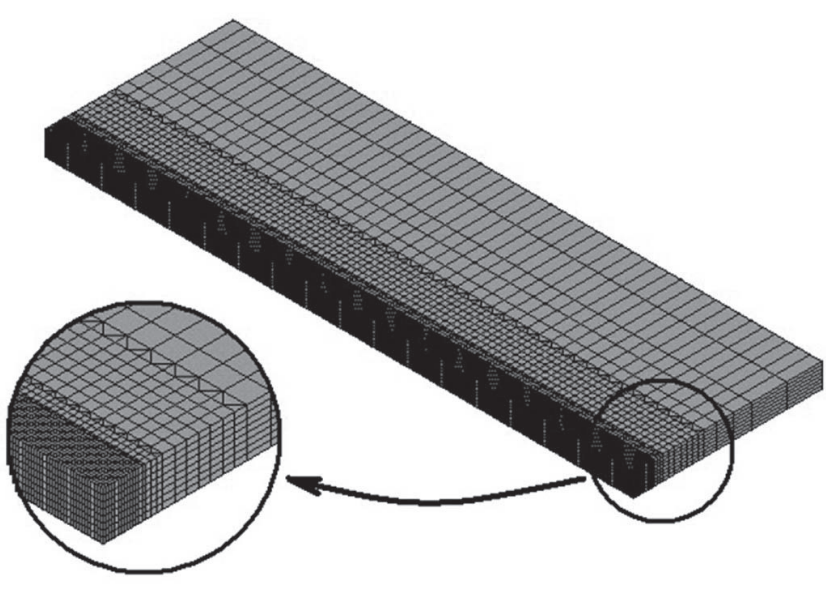

Figura 8 - Malla fina.

\subsection{Modelación térmica}

La modelación térmica fue ejecutada empleando el software de uso general ANSYS MULTIPHISYCS versión 12.0, aplicando la metodología de análisis térmico desarrollada por Pozo-Morejón [7, 8]. Dicha metodología fue enriquecida mediante la implementación del modelo de fuente de calor de doble elipsoide y el empleo de la entalpía como propiedad en el modelo, que permite considerar los cambios de fase, esto último analizado previamente en epígrafes 2 y 3 .

El modelo de fuente de calor de doble elipsoide fue implementado mediante subrutina desarrollada en el propio lenguaje APDL de ANSYS. Para cada paso de tiempo, dentro del tiempo de arco, en base a la posición del centro de la fuente se seleccionan los nodos que se encuentran dentro del doble elipsoide y a estos se les aplica la carga térmica calculada, mediante expresión 10 (o su equivalente para cuarto de elipsoide posterior), a través del comando "BF" y la etiqueta "HGEN". El recorrido a través de todos los nodos seleccionados, para el cálculo de la carga térmica, dentro de cada paso de tiempo y el salto de un paso de tiempo a otro se logra mediante lazos “*DO - *ENDDO”. Dentro de cada paso de tiempo se calcula el sistema mediante el comando "SOLVE" y finalizado el cálculo de cada uno se retira la carga térmica nodal mediante el comando "BFDELE". Finalmente se resuelve un último paso de tiempo, que equivale al tiempo de enfriamiento de la pieza hasta una temperatura cercana a la ambiente, con subpasos de tiempo de dimensión variable, acotada entre un valor mínimo igual a la magnitud de los pasos de tiempo de arco y un máximo, que fue establecido varias veces superior, en aras de reducir el tiempo de cálculo.

La subrutina que implementa el modelo de fuente de calor, mediante la aplicación de cargas nodales y el comando "BF", ha brindado buenos resultados, sin embargo estas cargas también se pueden aplicar sobre los elementos, en base a la ubicación de su centroide, mediante comando "BFE" y la misma etiqueta "HGEN", según reportan otros autores, como Malik [18].

Según metodología, fue establecido el método de solución "full" de "Newton Rapson", el valor de convergencia de la solución en $0,1{ }^{\circ} \mathrm{C}$ y la carga térmica fue aplicada a salto "stepped" 
en cada paso de tiempo [7, 8]. En este caso fue necesario elevar el número máximo iteraciones para alcanzar la convergencia hasta 60. Fue declarara como temperatura inicial de la placa 28 ${ }^{\circ} \mathrm{C}$. Sobre todas las áreas exteriores del modelo fue establecida una pérdida de calor por convección al medio de $10 \mathrm{~W} /\left(\mathrm{m}^{2} \times{ }^{\circ} \mathrm{C}\right)$, similar a la empleada por Depradeux [9], exceptuándose el área de simetría, donde no puede existir intercambio de calor. En el presente trabajo se han considerado las pérdidas de calor por radiación, con valores de interés a temperaturas cercanas a la de fusión, mediante el ajuste del valor de eficiencia de la fuente de calor $(\eta)$, como recomiendan varios autores $[6,7,8,19,20]$. Depradeux ha empleado en su modelación una eficiencia de la fuente de calor entre 70 y $80 \%$ [9], mientras que en este trabajo, luego de ajustes, se ha decidido emplear una eficiencia de $75 \%$, de manera que la potencia térmica que se introduce a los modelos es de $1125 \mathrm{~W}$ (calculada según expresión 7). Las propiedades termofísicas empleadas son las argumentadas en epígrafe 3.

Los pasos de tiempo mínimo se determinaron para cada tipo de malla de acuerdo a expresión 9, para una temperatura cercana a la de fusión $\left(1200{ }^{\circ} \mathrm{C}\right)$, los que fueron: malla gruesa $1,04 \mathrm{~s}$, media $0,26 \mathrm{~s}$ y fina $0,07 \mathrm{~s}$. Con vistas a analizar la influencia de esta variable, y siguiendo recomendaciones expuestas en el epígrafe 2.3 , se eligen pasos de tiempo de 2,$5 ; 5 ; 10 ; 15$ y 40 $\mathrm{s}$, que representan saltos de la fuente de calor de 0.17 veces su longitud, hasta 2,73 veces y cumplen con condición 11 .

Las dimensiones utilizadas en el modelo de doble elipsoide fueron establecidas cercanas a la dimensión de la sección transversal de la ZF, según macrografía de Depradeux [9], tomándose $\mathrm{a}=3.25 \mathrm{~mm} \mathrm{y} \mathrm{b}=4.1 \mathrm{~mm}$. Fueron asumidos $\mathrm{c}_{\mathrm{f}}=$ $3.25 \mathrm{~mm} \mathrm{y} \mathrm{c}_{\mathrm{b}}=6,5 \mathrm{~mm}$, ya que para estas dimensiones no se poseen datos experimentales. Fue establecida por tanteo una duración de $4000 \mathrm{~s}$ para el paso de tiempo final de enfriamiento.

\subsection{Resultados y discusión}

Primeramente fue analizada la convergencia de los resultados para las distintas mallas (gruesa, media y fina) con el empleo de un paso de tiempo de 2,5 s. Fue demostrado que para las tres mallas prácticamente las curvas de los ciclos térmicos se sobreponen una sobre otra, de manera que el mayor error relativo se presenta en el nodo sobre el eje de la soldadura (x $=0$ ) en sección ST1, en el pico de máxima temperatura, con apenas $1,2 \%$ de error relativo (figuras 9 y 10). A continuación fue realizada la superposición de las curvas que definen el perfil de la zona fundida determinadas con los tres tamaños de malla, fue calculada la dimensión del semiancho y la penetración, determinándose que el mayor error relativo entre estas fue de 3,8 \% (figura 11). Es evidente que el perfil de la zona fundida determinado por la malla basta difiere en cuanto a configuración del experimental, debido a que solo hay dos elementos enteros sobre el semieje del elipsoide. Los perfiles calculados mediante las mallas fina y media prácticamente coinciden. Como isoterma que delimita la frontera de la ZF se tomó la temperatura de 1475 ${ }^{\circ} \mathrm{C}$ (temperatura media entre " $\mathrm{T}_{1}$ " $\mathrm{y}$ " $\mathrm{T}_{\mathrm{s}}$ " para esta aleación). De esta manera se puede afirmar que los resultados de las tres mallas convergen, por lo que son adecuadas para la corrida térmica. Por supuesto, si los resultados de interés fueran en la

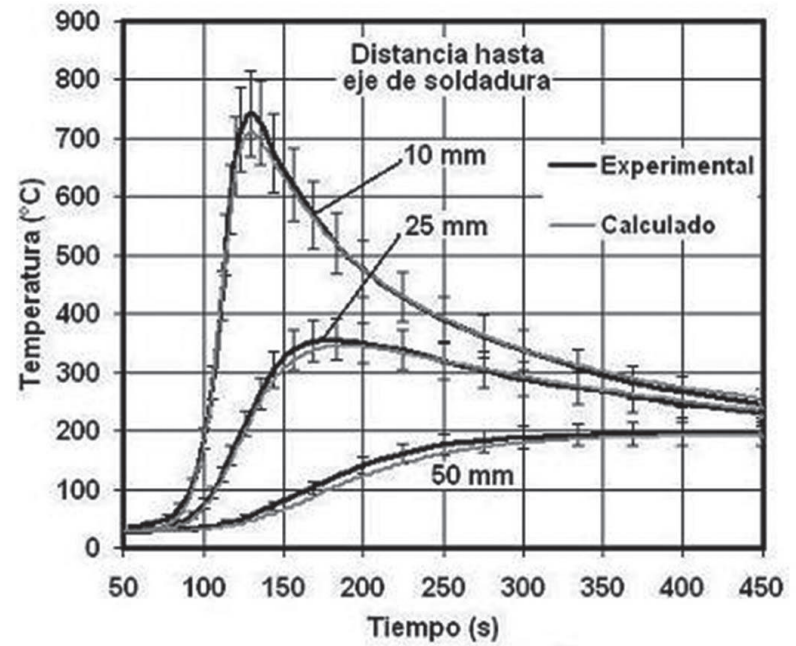

Figura 9 - Ciclos térmicos de puntos situados a 10,25, $50 \mathrm{~mm}$ del eje de la soldadura, sección ST1, cara superior de placa, paso de tiempo 2,5 s (coinciden mallas fina, media y gruesa). Barras de error $\mathrm{a} \pm 10 \%$.

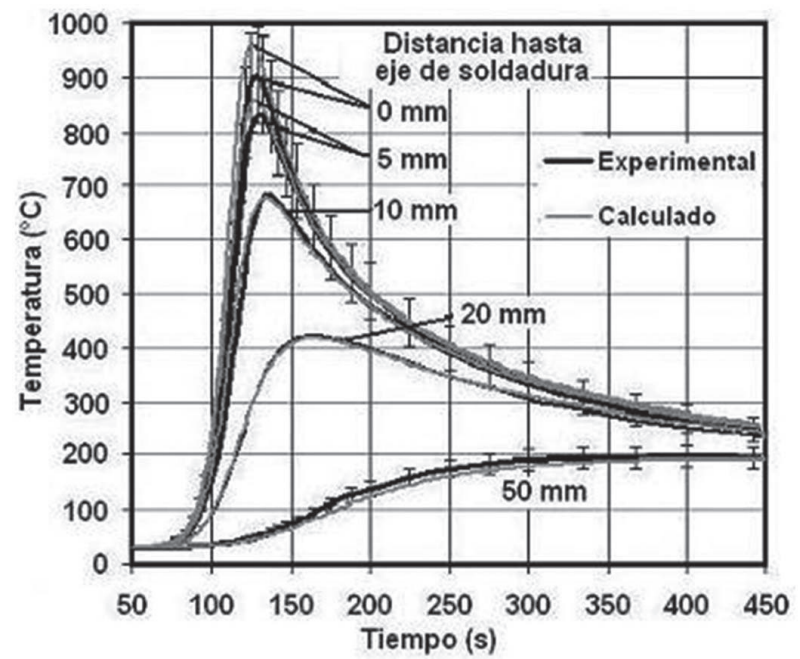

Figura 10 - Ciclos térmicos de puntos situados a $0,5,10,20$, $50 \mathrm{~mm}$ del eje de la soldadura, sección ST1, cara inferior de placa, paso de tiempo de 2,5 $\mathrm{s}$ (coinciden mallas fina, media y gruesa). Barras de error a $\pm 10 \%$.

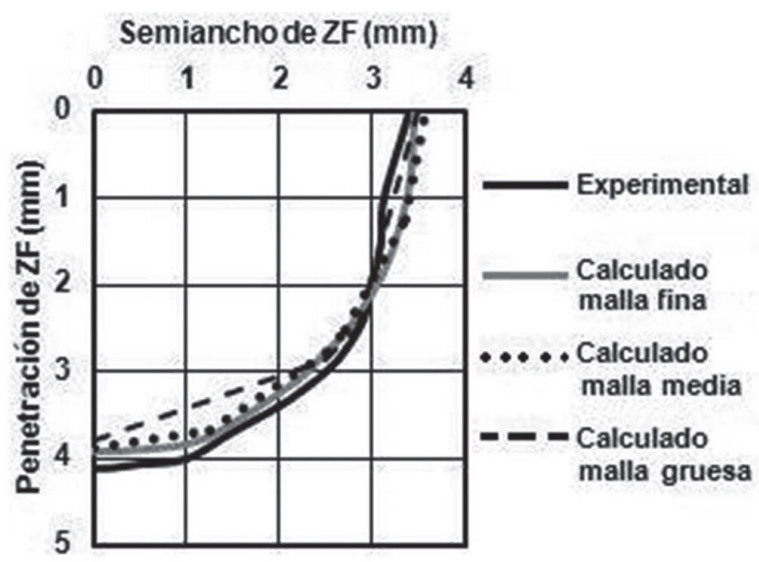

Figura 11 - Comparación entre zona fundida experimental (macrografía en ST1) y calculadas para diferentes tamaños de malla, con paso de tiempo de $2.5 \mathrm{~s}$. 
zona cercana a la soldadura, se deben preferir las mallas fina y media que garantizan la mayor precisión, mientras que si se pretende realizar con posterioridad una corrida mecánica la mejor selección sería la malla gruesa porque reduce grandemente el tiempo computacional.

En las figuras 9 y 10 se comparan, además, los resultados de los modelos respecto a los experimentales para la cara superior e inferior de la placa. Se aprecia una alta coincidencia entre las curvas experimentales y las calculadas, con un por ciento de error relativo $<5 \%$ para las distancias de 10 y $25 \mathrm{~mm}$ y $<$ $10 \%$ para la distancia de $50 \mathrm{~mm}$ en la cara superior (figura 9). Para la cara inferior en las distancias de 0, 5, 10, $25 \mathrm{~mm}$ el error es $<5 \%$ y $<10 \%$ para la distancia de $50 \mathrm{~mm}$ (figura 10). Al comparar las dimensiones del semiancho y la penetración de la ZF experimentales y calculadas se obtiene que el error relativo máximo es de alrededor de $6 \%$ para las mallas fina y media, y de alrededor del $10 \%$ para la basta (tabla 1). De esta manera se ha validado la metodología de análisis térmico enriquecida, que implementa el modelo de doble elipsoide de Goldak en un sistema de coordenadas cartesiano, ya que fue demostrado conduce a resultados de dimensiones y configuración del baño, así como ciclos térmicos para puntos situados a diferentes distancias con un error inferior al $10 \%$. Fue demostrado además que las propiedades termofísicas asumidas en epígrafe 3 son correctas.

Tabla 1 - Error calculado respecto a experimental para dimensiones de la zona fundida con diferentes tamaños de malla (macrografía en ST1). Corrida con paso de tiempo de $2.5 \mathrm{~s}$

\begin{tabular}{|c|c|c|c|c|}
\hline Corrida & $\begin{array}{c}\text { Semiancho } \\
(\mathbf{m m})\end{array}$ & $\begin{array}{c}\text { Error } \\
\mathbf{( \% )}\end{array}$ & $\begin{array}{c}\text { Profundidad } \\
(\mathbf{m m})\end{array}$ & $\begin{array}{c}\text { Error } \\
\mathbf{( \% )}\end{array}$ \\
\hline Experimental & 3.4 & 3.1 & 3.9 & 6.02 \\
\hline $\begin{array}{c}\text { Calc. Malla } \\
\text { fina }\end{array}$ & 3.5 & 2.03 & 3.8 & 6.26 \\
\hline $\begin{array}{c}\text { Calc. Malla } \\
\text { media }\end{array}$ & 3.5 & 2.9 & 3.7 & 9.63 \\
\hline $\begin{array}{c}\text { Calc. Malla } \\
\text { gruesa }\end{array}$ & 3.4 & 0.58 & & \\
\hline
\end{tabular}

Para analizar la influencia del paso de tiempo fueron realizadas corridas con diferentes valores de este $(2,5 ; 5 ; 10 ; 15$ y $40 \mathrm{~s}$ ), empleando la malla media. Es evidente que las curvas de ciclos térmicos para el paso de tiempo de $40 \mathrm{~s}$, que equivale a un salto de la fuente de 2,73 veces su longitud, presentan una configuración burda y se alejan del resto y de los resultados experimentales, atrasándose en demasía en el tiempo, con un error relativo superior al $10 \%$ (figuras 12 y 13); esto se corresponde con lo planteado por Goldak ya que este paso de tiempo se acerca al salto de 3 veces la longitud de la fuente, que según este autor brinda pobres resultados, como fue referido en epígrafe 2.3. Téngase en cuenta que en este caso el calor se aplica sobre el modelo de elementos finitos en zonas distanciadas, que no se solapan unas con otras, por lo que hay una gran cantidad de elementos en la zona de la soldadura que no reciben calor, lo que se aleja del fenómeno real.
Al analizar el resto de las curvas de ciclos térmicos, en las figuras 12 y 13, se observa que en la medida que el paso de tiempo aumenta, aumenta el retraso de las mismas respecto a los resultados experimentales y disminuye la temperatura máxima que estas alcanzan. Este resultado es lógico ya que en la medida que en el modelo el paso de tiempo se hace mayor, la posición de la fuente a lo largo de su eje de desplazamiento se retrasa, por lo que el calor demora más en llegar a cualquier nodo aledaño a la soldadura. La disminución de la temperatura máxima alcanzada se justifica, ya que en la medida que el paso de tiempo aumenta, las zonas donde se aplica el calor están menos solapadas, por lo que la influencia del calor que se transmite por conducción hasta los nodos aledaños a la soldadura desde la zona que antecede y que sucede es inferior que cuando el paso de tiempo es menor. Este comportamiento no se cumple para el caso del paso de tiempo de $40 \mathrm{~s}$ ya que aquí prima el gran tiempo que la fuente está aplicando calor cerca de la sección transversal donde se colocan los termopares (ST1).

Del análisis de las figuras 12 y 13 se puede concluir que para los pasos de tiempo de $2,5 \mathrm{~s}$ y de $5 \mathrm{~s}$ (donde la fuente de calor salta 0,17 y $1 / 3$ veces su longitud respectivamente) las curvas de temperatura se mantienen relativamente cercanas entre si y respecto al resultado experimental, siempre dentro del $10 \%$ de error relativo, concordando con lo establecido por Goldak y analizado en epígrafe 2.3. Para los pasos de $10 \mathrm{~s}$ y $15 \mathrm{~s}$ (donde la fuente salta $2 / 3$ y una vez su longitud respectivamente) las curvas, aunque se mantienen relativamente cercanas a las anteriores, exceden el error relativo del $10 \%$ en el estadío de calentamiento, mientras que garantizan buena precisión durante el enfriamiento.

En cuanto a las dimensiones del baño (figura 14) es claro que el paso de tiempo de $40 \mathrm{~s}$ se aleja del resto de los pasos, aumentando dichas dimensiones producto del exceso de tiempo en que se aplica el calor sobre una misma zona. Se justifica que la dimensión de la zona fundida para el paso de tiempo de 2,5 s sea algo mayor que para los pasos de $5 \mathrm{~s}$ y $10 \mathrm{~s}$, ya que en este caso se impone el extremo solapamiento de las zonas donde se aplica el calor, de manera que este se transmite por conducción desde la zona que la antecede y sucede.

A modo de resumen, a partir de los resultados analizados se puede inferir que el paso de tiempo elegido para las corridas durante una simulación de soldadura mediante elementos finitos se debe alejar por encima del calculado por la expresión 9, con vistas a reducir los tiempos de cómputo. Los pasos de tiempo aquí empleados de $2,5 \mathrm{~s}$ y $5 \mathrm{~s}$, que garantizan saltos inferiores a la recomendación de Goldak (de que el paso de tiempo garantice saltos de aproximadamente $1 / 2$ veces la longitud de la fuente), brindan resultados precisos, en concordancia con dicho autor.

Es conocida la reducción de los tiempos de cálculo que estos pasos de tiempo provocan, sobre todo en la corrida mecánica, lo que es muy importante si se persigue llegar hasta su ejecución. Sería de interés para futuros trabajos corroborar la precisión de los resultados de tensiones y desplazamientos que alcanzan estos pasos de tiempo en una corrida mecánica, aunque esto queda fuera de los objetivos propuestos en esta ocasión. 


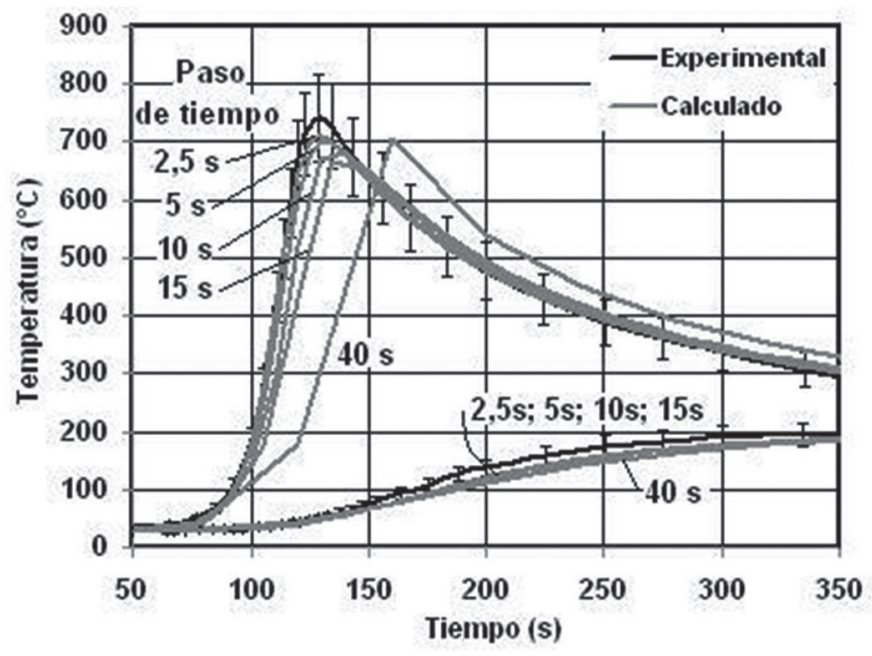

Figura 12 - Influencia del paso de tiempo sobre el ciclo térmico para puntos en $\mathrm{x}=10 \mathrm{~mm} \mathrm{y} x=50 \mathrm{~mm}$, sobre cara superior.

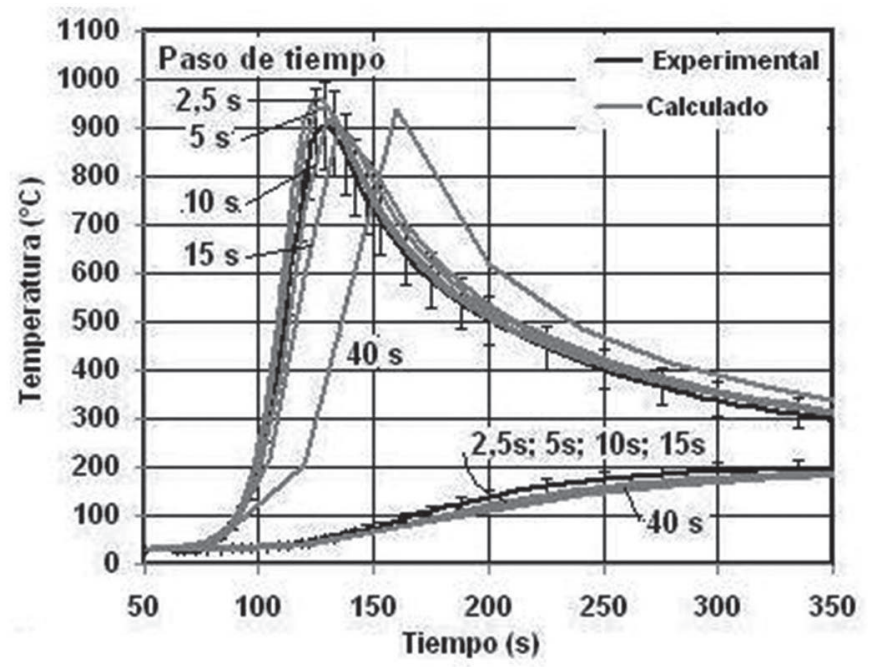

Figura 13 - Influencia del paso de tiempo sobre el ciclo térmico para los puntos con $\mathrm{x}=0 \mathrm{~mm} \mathrm{y} x=50 \mathrm{~mm}$, en la cara inferior.

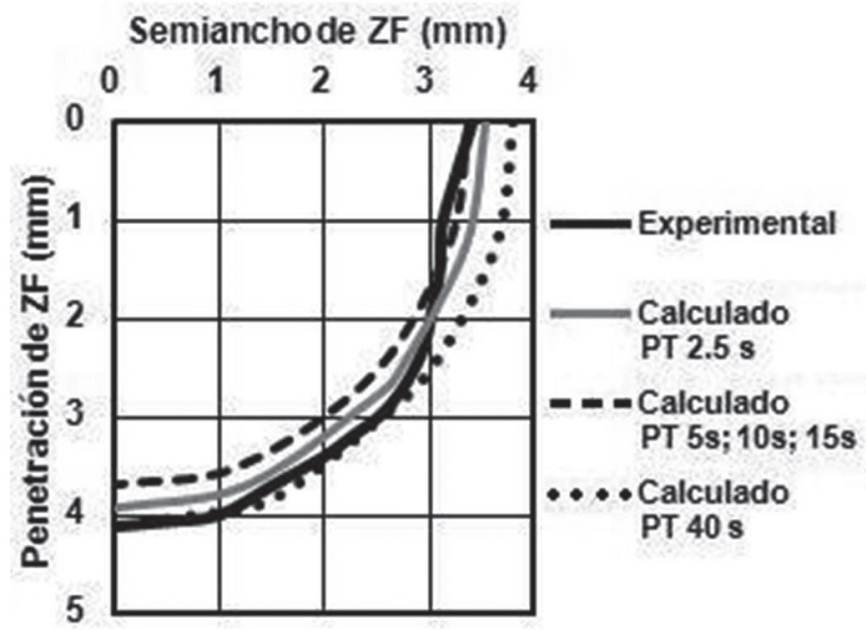

Figura 14 - Influencia del paso de tiempo sobre la forma y dimensiones de la ZF.

\section{Conclusiones}

1 - Los resultados obtenidos en las simulaciones de soldadura mediante el AEF, de temperaturas de ciclos térmicos para puntos situados a diferentes distancias y de dimensiones del baño, manifiestan una alta correlación con los experimentales, lo cual evidencia:

- La adecuada selección de las propiedades termofísicas, de conductividad térmica y entalpía, para el acero AISI $316 \mathrm{~L}$,

- La validez de la metodología para la ejecución de un análisis térmico mediante ANSYS, así como de la subrutina en lenguaje APDL, que implementa el modelo de doble elipsoide de Goldak, en un sistema de coordenadas cartesiano.

2 - Se corrobora que el paso de tiempo elegido para las corridas térmicas durante una simulación de soldadura mediante AEF se debe alejar por encima del calculado por la expresión 9, con vistas a no aumentar en exceso el tiempo de cálculo, sobre todo para las mallas más finas, pero sin sobrepasar el valor que provoca un desplazamiento de la fuente de $1 / 3$ veces la longitud de la fuente. Se comprueba además que en la medida que el paso de tiempo aumenta, aumenta proporcionalmente el retraso de las curvas de temperaturas calculadas respecto a las experimentales.

3 - La metodología enriquecida de modelación de soldaduras constituye un paso de avance hacia la posibilidad de estudio de diversos fenómenos propios del proceso de soldeo, empleando un software de AEF de uso general, y la solución de múltiples problemas industriales, a tono con los esfuerzos que se desarrollan en este campo a nivel mundial.

\section{Bibliografía}

[1] ZHU, X. K., CHAO, Y. J. Effects of temperature-dependent material properties on welding simulation. Computers and Structures 80, p. 967-976, 2002.

[2] FRANCIS, J. D. Welding simulations of aluminum alloy joints by finite element analysis. Tesis en opción del grado de Master of Science en Ingeniería Aeroespacial, Faculty of the Virginia Polytechnic Institute and State University. 2002.

[3] YAJIHAN, L., WANG, J. Finite Element analysis of Residual Stress in the weld Zone of a high Strength Steel. Key Laboratory of Liquid structural and heredity of Materials. Ministery of Education Shandong University, Jinan 250061. China. Indian Academy of Science. Vol 97, $\mathrm{N}^{\circ}$ 2, abril, 2004.

[4] BEZERRA, A.C. Simulação Numérica da Soldagem com Aplicação à Caracterização do Comportamento Dinâmico de Estruturas Soldadas. Tesis de Doctorado, Universidad Federal de Uberlândia, Uberlândia. Brasil. 2006.

[5] YU, H., SHOUJU, L., YINGXI, L. Finite Element Simulation for Residual Stresses in Welding Process. Engineering Materials Vol. 353-358, p. 1915-1918, 2007.

[6] RAMOS MORALES, F., DUFFUS SCOTT, A., RODRÍGUEZ PÉREZ, M., DÍAZ CEDRÉ, E., POZO MOREJÓN, J. A., MIGUEL ORIA, J. V. Modelo de elementos finitos para determinar el campo de temperatura en una costura de filete. Ingeniería, Revista Académica de la FI-UADY, 11-3, p. 
33-42, ISSN: 1665-529X. 2007.

[7] POZO MOREJÓN, J. A. Metodología para el establecimiento de procedimientos de soldadura en aleaciones de aluminio al magnesio mediante la modelación por elementos finitos. Tesis doctoral. Universidad Central "Marta Abreu" de Las Villas. Santa Clara. Cuba. 2008.

[8] POZO MOREJÓN, J., GARCÍA, J., RAMOS, F., GARCÍA, Y., CRUZ, A., DÍAZ, E., DUFFUS, A., Metodología de modelación mediante ANSYS de la historia térmica, tensiones y deformaciones de soldadura. Revista de la Facultad de Ingeniería U.C.V., Vol. 24, N 1, pp. 13-26. 2009.

[9] DEPRADEUX, L. Simulation Numerique du Soudage - Acier 316L. Ecole Doctorale des Sciences de L'Ingénieur de Lyon (MEGA). Francia, L'Intitut National des Sciences Appliquees de Lyon. 2004.

[10] INCROPERA, F. P., DE WITT, D. P. Fundamentals of Heat and Mass Transfer. Tercera Edición, John Wiley. 1990.

[11] GOLDAK, J. A., MEHDI AKHLAGHI. Computational welding mechanics. New York. Springer Science+Business Media, Inc., 2005.

[12] NGUYEN, N., MAI, Y., SIMPSON, S. \& OHTA, A. Analytical Approximate Solution for Double Ellipsoidal Heat Source in Finite Thick Plate. Welding Research. 2004.

[13] CRONJE, M. Finite element modeling of shielded metal arc welding. Tesis presentada en opción al grado de Master of Science en Ing. Mecánica. Department of Mechanical Engineering. Stellenbosch University. Diciembre. 2005.

[14] ANSYS INC. Tutorial de ANSYS MULTIPHYSICS versión 12.0 .

[15] BARAN, N. M. Finite Element Analysis on Microcomputers. McGraw - Hill Inc- Hill Inc., United States, 1988. 140 p. 1998.

[16] GOLDAK, J., ASADI, M., GARCIA, R. Why power per unit length of weld does not characterize a weld. Computational Materials Science. Vol. 48, No. 2, p. 390-401, 2010.

[17] SUN, J. Modeling and Finite Element Analysis of Welding Distortions and Residual Stresses in Large and Complex Structures. Tesis de Doctorado. The Pennsylvania State University, Pennsylvania, USA. 2005.

[18] MALIK, A., EJAZ, M., ULLAH, N. Numerical Simulation of Arc Welding Investigation of various Process and Heat Source Parameters. Failure of Engineering Materials \& Structures, Code 30, 2007.

[19] WEST, S. L. Modeling of residual stress mitigation in austenitic stainless steel pipe girth weldment. International Conference on Modeling and Control of Joining Processes. Oregon Graduate Institute. 1993.

[20] ARMENTANI, E. E., R.; SEPE, R. Valutazione numerica delle tensioni residue in processi di saldatura GMAW. Associazione Italiana per l'Analisi delle Sollecitazioni (AIAS). XXXV Convegno Nazionale.13-16 Settembre 2006, Università Politecnica delle Marche. 2006. 